\title{
Rationales for indicator condition-based HIV testing data from the Hospital for Infectious Diseases in Warsaw - one-year observation
}

\author{
Karolina A. Pyziak-Kowalska ${ }^{1,2}$, Justyna Kowalska ${ }^{2,3}$, Andrzej Horban ${ }^{1,2}$ \\ ${ }^{1}$ Emergency Department, Hospital for Infectious Diseases, Warsaw, Poland \\ ${ }^{2}$ Department for Adults Infectious Diseases, Medical University of Warsaw, Warsaw, Poland \\ ${ }^{3}$ HIV Out-Patient Clinic, Hospital for Infectious Diseases, Warsaw, Poland
}

\begin{abstract}
Introduction: The European study HIDES (HIV Indicator Disease Across Europe Study) has shown that indicator condition (IC)-based HIV testing may increase the number of detected HIV infections among patients with a given medical conditions. Currently routine HIV testing for IC is not covered by public healthcare in Poland, which may delay or miss the opportunity for HIV diagnosis. The aim of the study was to evaluate HIV testing patterns among patients presenting with specific IC-ongoing mononucleosis-like illness (mononucleosis) in emergency departments (ED).
\end{abstract}

Material and methods: We performed retrospective analysis of patients with mononucleosis referred from ED to hospital departments for further diagnostics within the past 12 months.

Results: In total 173 patients were consulted in ED with mononucleosis, that is 94 men and 79 women, with median age of 26 years. Seventy-two $(41.6 \%)$ patients were admitted to hospital, among whom 54 (75\%) were offered an HIV test and all expressed consent. Four patients (5.5\%) were diagnosed with HIV, referred to an HIV clinic, and linked to care. According to analyses, $68 \%$ of patients missed the opportunity for HIV testing, 58\% in ED due to lack of such healthcare program, and $10 \%$ of unknown reason. With the presented rate, this translated to eight HIV patients who may still remain unaware of HIV infection.

Conclusions: The rate of HIV diagnosis among patients hospitalised due to mononucleosis was high, confirming the benefit of routine testing of this group of patients. Standards of care for mononucleosis should include routine HIV testing, which needs additional financing and attention from public healthcare representatives and other stakeholders.

HIV AIDS Rev 2017; 16, 3: 191-194 DOI: https://doi.org/10.5114/hivar.2017.70756

Key words: indicator conditions, optimisation, mononucleosis-like syndrome, missed opportunity for HIV testing, routine HIV testing.

Address for correspondence: Dr Karolina A. Pyziak-Kowalska, Emergency Department, Hospital for Infectious Diseases, Warsaw, 37 Wolska St., 01-201 Warsaw, Poland,

e-mail: karolina.pyziak@gmail.com
Article history:

Received: 18.12.2016

Received in revised form: 31.07.2017

Accepted: 04.10.2017

Available online: 10.10.2017
International Journal of HIV-Related Problems

HIV \& AIDS

R e v i e w 


\section{Introduction}

Despite the enormous progress in the treatment of the HIV/AIDS-diagnosed patients and the common access to highly effective antiretroviral treatment, an increase in new HIV infections in Europe can still be observed [1]. Moreover, it is estimated that in Europe, among 2.3 million HIV-infected persons, one in three is unaware of the fact of being infected [2].

Poland belongs to the countries with low prevalence of HIV infection, but in the last five years up to 1200 new HIV cases are diagnosed each year, which is more than double the increase in relation to the previous years [3]. It is estimated that in Poland even 70\% of HIV-infected persons do not know about their status, and these persons are not included in the statistics [4].

The subsequent worrying aspect is the persistent high percentage of patients diagnosed at a late stage of the disease [5]. It is particularly noticeable in the Central and Eastern Europe [6].

Therefore, it is believed nowadays that the key element in the control of the HIV epidemic is the optimisation of testing for HIV. So far, the carried out European studies have shown that the testing model, based on the routine indicator condition-guided HIV testing, is effective in the reality of clinical practices and the centres that do not specialise in the diagnostics and treatment of HIV infection [6-8].

Since May 2015 in the Hospital of Infectious Diseases in Warsaw an international project regarding the optimisation of the testing for HIV, namely the Optimisation of Testing and Linkage to Care (OptTEST Project) is carried out [9]. The aim of the project is the reduction of the number of persons with non-diagnosed HIV infection and persons with the HIV diagnosis [10]. The study aims at the promotion of the routine performance of the tests for the HIV infection among all persons with specific indicator conditions, the prevalence of which in the population amounts to $>0.1 \%$ [9]. The proposed modern testing strategy is based on the results of the European project HIDES (HIV Indicator Disease Across Europe Study), which has shown the effectiveness of the testing of all patients with the specific indicator conditions $[11,12]$.

One of the crucial indicator condition with the highest prevalence - 5.3 (95\% CI: 3.7-6.9\%) [13] is the mononucleosis-like syndrome which may be the first and only clinical manifestation of the acute retroviral infection [9]. The performance of the HIV test of patient presenting with the indicator condition - with the mononucleosis-like syndrome, gives a possibility of the detection of the HIV infection at an early stage of a disease and fast provision of the specialist care of an ill person [14]. It is worth stressing that the relation between an early diagnosis and the beginning of the antiretroviral treatment and the reduction of the AIDS morbidity and the prolongation of the HIV-infected persons' survival was documented. An additional, relevant aspect of the treatment is the reduction of the risk of the transmission of the virus $[15,16]$.
Therefore, the aim of our study was the retrospective review of the existing practice in the area of the testing for HIV in the mononucleosis-like syndrome.

\section{Material and methods}

We conducted retrospective analysis of the medical records of patients who were consulted at the Emergency Department of the Hospital for Infectious Diseases in Warsaw. We have reviewed patients records within a period of 12 months (01.05.2014-30.04.2015).

The patients were eligible for the analyses if being 18 years or older and who had mononucleosis-like syndrome diagnosed by the infectious diseases specialist. Data collected included age, sex, medical indications for hospitalisation, and the information whether the patient had a test for the HIV performed. The diagnosis of mononucleosis like-syndrome was confirmed by the medical history, examination of the patient, and by the presence of more than $10 \%$ of atypical lymphocytes.

\section{Results}

In total, the medical records of 173 patients with mononucleosis-like illness were analysed. Within the test group there were 94 (54\%) men and 79 (46\%) women. The median age was $26(18-43)$ years, including 26 for women and 27 for men.

Due to the severe course of the disease, $72(41,6 \%)$ patients were admitted to the hospital. Within this group of patients there were 39 (54.1\%) men and 33 (45.9\%) women. During their stay at the hospital, $54(75 \%)$ patients were offered HIV tests and all expressed consent; among them $32(82 \%)$ men and $22(66.7 \%)$ women, with median age of 26.5 (18-43) years. In the medical documentation of the hospitalised patients 18/72 (25\%) who were not offered a test, no information was found as to why a part of the patients did not have the test for the HIV performed.

Four (5.5\%) patients from 72 hospitalised were diagnosed with HIV infection - all patients with a newly-detected HIV infection were men who have sex with men (MSM), the median age of the patients was $24(20-41)$ years, the median of the CD4+ lymphocytes count was 564.5 (min. 347, max. 855) cells per microliter. All patients were linked to and received HIV specialist care at the Outpatient Clinic in Warsaw and started the antiretroviral treatment in a period of one to six months.

Figure 1 shows lost opportunities for HIV testing in medical settings.

\section{Discussion}

The percentage of the patients with the newly detected HIV infection who were hospitalised due to the mononucleosis-like syndrome in the Hospital for Infectious Diseases in Warsaw was high, and it amounted to $5.5 \%$. Undoubtedly, it 


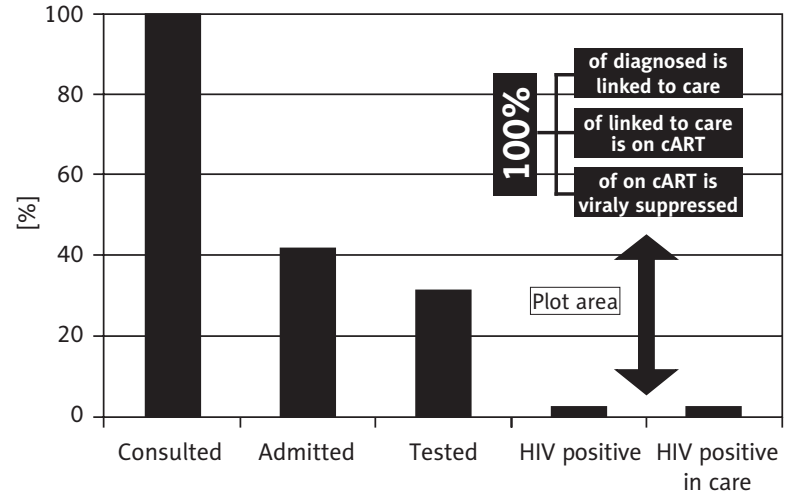

Figure 1. Lost opportunities for HIV testing in medical settings

confirms the benefits arising from the routine testing of all patients with mononucleosis-like syndrome [17]. Currently, the tests for the detection of HIV are not routinely performed in hospitals' emergency departments, in Poland or in the rest of Europe. A fact of a relevant significance is that two out of three patients who sought care at the Hospital for Infectious Diseases in Warsaw due to the mononucleosis-like syndrome did not have the test for HIV infection performed. A direct cause is the lack of the nationwide programme of the routine testing of all patients with the specific indicator condition, for example persons with mononucleosis-like syndrome. As a result, according to our estimations, at least eight patients who may be infected with HIV remain unaware of the fact of being infected, which not only leads to the progress of the disease but, with the lack of the awareness of the infection, also leads to the transmission of the virus to other sexual partners. It is indispensable to undertake other actions aimed at the increase of the initiatives for the early diagnosis of HIV infection based on the specific indicator conditions with HIV prevalence of more than $0.1 \%$.

A lack of national investment in HIV prevention results in inadequate or absent possibilities to stop the HIV epidemic in Poland. Therefore, this proposed innovative approach, indicator condition-guided HIV testing, might be an additional element of overall comprehensive national testing strategy. This strategy should be adopted not only by Polish AIDS Society guidelines, but also by other scientific and clinical associations, for example for general practitioners.

At the same time, analyses from Poland show that, independent of transmission mode, sexual activity, and risk behaviours, starting antiretroviral treatment proves to be always cost-effective [18].

A late HIV infection diagnosis, when already the AIDS defining diseases occur, constitutes a relevant problem in Poland [19-21]. Nowadays, the tests for the HIV infection are performed in Poland in two ways. First of all, by the carrying out of the programme of the anonymous testing in the Voluntary Counselling and Testing Centres (VCT) (Polish abbreviation: PKD) based on the analysis of the risk factors [22]. This system detects around $1.3 \%$ of new HIV infections which indicates its high effectiveness [23]. Moreover, in the VCTs there are tested persons having risky behaviours, thus the system enables an early detection of an infection and limits its transmission.

The second form of testing is the medical testing, which is based on the HIV testing offered to patient in the public healthcare settings [24]. Taking into consideration the high number of persons with the late stage diagnosis of the disease, the effectiveness of such conduct seems to be insufficient [25]. Retrospective case analyses prove lost opportunities for HIV testing during past medical care. Even patients at risk are not offered HIV testing by medical personnel.

The medical care system for HIV-diagnosed patients currently functioning in Poland guarantees access to free antiretroviral treatment. In accordance with the newest guidelines of the PTN AIDS and EACS, each patient with the confirmed HIV infection and the readiness to take up treatment should begin it without undue delay $[24,26]$. The percentage of the persons receiving effective therapy and persons with its full effectiveness in Poland is high, yet the percentage of the persons who seek medical care after the HIV infection diagnosis remains unsatisfying $[27,28]$.

As was shown in the Test and Keep in Care (TAK) project, as many as $40 \%$ of persons diagnosed in the PKDs may not seek medical care.

Late diagnosis of the HIV infection is associated with the increased morbidity and mortality, worse response to the applied treatment, and significantly increased costs of care and treatment which causes enormous social and financial losses [16].

Therefore, there exist many benefits of diagnosing HIV at an early stage [10], which means that the key strategy of public health in Poland seems to be the innovative attitude towards medical testing and the increase in seeking medical care by persons with the confirmed infection [29].

The inspiration is the 90-90-90 strategy proposed by the UNAIDS, which stipulates focus on the realisation of the three postulates. The first one stipulates that by 2020 $90 \%$ of HIV infected persons will know their serological status. Among the diagnosed persons $90 \%$ will receive treatment, and among them $90 \%$ will be treated effectively (which means they will have undetectable viral load) [30]. In the Polish reality achievement of the first $90 \%$ seems to be the basic problem in the realisation of such strategy, thus there is an effective testing strategy and the high percentage in linkage to specialist medical care.

As far as the results obtained from the study carried out, it is worth stressing the key role which the promotion of the routine testing of patients with the mononucleosis-like syndrome has for public health. It should be taken into consideration how to increase funds for the diagnostics so that the tests for the HIV infection could be performed on a routine basis, not only in patients with mononucleosis-like syndrome demanding hospitalisation but also those who seek medical care in hospital Emergency Departments and General Practitioners (GPs). Currently, in the Emergency Department of the Hospital for Infectious Diseases 
in Warsaw the realisation of a subsequent scientific project, aimed at the performance of HIV testing among patients with mononucleosis-like syndrome admitted to this unit in the last two years, was commenced.

\section{Conclusions}

Our study confirms the high effectiveness of testing for HIV of all patients with mononucleosis-like syndrome diagnosed in the Hospital for Infectious Diseases in Warsaw. The scientific research should be continued, which will verify in general the benefits of routine indicator condition-based HIV testing for patients with the specific indicator conditions such as pneumonia or a newly diagnosed HCV infection [17, 31].

\section{Conflict of interest}

The author's declared no potential conflicts of interest with respect to the research, authorship, and/or publication of this article.

\section{References}

1. HIV AIDS surveillance in Europe 2014. Available at: http://ecdc. europa.eu/en/publications/Publications/hiv-aids-surveillance-inEurope-2014.pdf

2. HIV Indicator Conditions: Guidance for Implementing HIV Testing In Adults In Health Care Settings. Available at: http://hiveurope. eu/Portals/0/Guidance.pdf.pdf

3. HIV/AIDS Morbidity and Mortality in Poland. Available at: http:// wwwold.pzh.gov.pl/oldpage/epimeld/hiv_aids/Ryc_1.jpg

4. Schedule of implementation of the National Program for HIV and AIDS prevention prepared for 2012-2016. The National AIDS Centre, 2012.

5. Mocroft A, Lundgren JD, Sabin ML, et al. Risk factors and outcomes for late presentation for HIV-positive persons in Europe: results from the Collaboration of Observational HIV Epidemiological Research Europe Study (COHERE). PLoS Med 2013; 10: e1001510.

6. Reekie J, Kowalska JD, Karpov I, et al. Regional differences in AIDS and non-AIDS related mortality in HIV-positive individuals across Europe and Argentina: the EuroSIDA study. PLoS One 2012; 7: e41673.

7. Smith CJ, Ryom L, Weber R, et al. Trends in underlying causes of death in people with HIV from 1999 to 2011 (D:A:D): a multicohort collaboration. Lancet 2014; 384: 241-248.

8. Baggaley R. HIV for non-HIV specialists, Diagnosing the undiagnosed 2008. Available at: http://www.medfash.org.uk/publications/ documents/HIV_for_non_HIV_specialists.pdf

9. Sullivan AK, Raben D, Reekie J, et al. Feasibility and effectiveness of indicator condition-guided testing for HIV: results from HIDES I (HIV indicator diseases across Europe study). PLoS One 2013; 8: e52845.

10. Gullón A, Verdejo J, de Miguel R, et al. Factors associated with late diagnosis of HIV infection and missed opportunities for earlier testing. AIDS Care 2016; 28: 1296-1300.

11. Rayment M, Thornton A, Mandalia S, et al. HIV testing in non-traditional settings - the HINTS study: a multi-centre observational study of feasibility and acceptability. PLoS One 2012; 7: e39530.

12. Agustí C, Montoliu A, Mascort J, et al. Missed opportunities for HIV testing of patients diagnosed with an indicator condition in primary care in Catalonia, Spain. Sex Transm Infect 2016; 92 387-392.
13. HIDES: Indicator Diseases across Europe Study. Available at: http:// newsite.hiveurope.eu/Ongoing-Projects/HIDES

14. Hsu DT, Ruf M, O'Shea S, et al. Diagnosing HIV infection in patients presenting with glandular fever-like illness in primary care: are we missing primary HIV infection? HIV Med 2013; 14: 60-63.

15. UK Collaborative HIV Cohort (UK CHIC) Steering Committee; Sabin CA, Schwenk A, Johnson MA, et al. Late diagnosis in the HAART era: proposed common definitions and associations with mortality. AIDS 2010; 24: 723-727.

16. Cohen MS, Chen YQ, McCauley M, et al. Prevention of HIV-1 infection with early antiretroviral therapy. N Engl J Med 2011; 365: 493-505.

17. Raben D, Mocroft A, Rayment M, et al. Auditing HIV Testing Rates across Europe: Results from the HIDES 2 Study. PLoS One 2015; 10: e0140845.

18. Kowalska JD, Wójcik G, Rutkowski J, et al. Modelling the cost-effectiveness of HIV care in Poland shows clear benefit while transmission risk is considered in the calculation Poster Nr P327 presented at the HIV Drug Therapy Congress, Glasgow 2016.

19. Podlasin RB, Wiercinska-Drapalo A, Olczak A, et al. Opportunistic infections and other AIDS-defining illnesses in Poland in 20002002. Infection 2006; 34: 196-200.

20. Zalewska-Schonthaler N, Schonthaler-Humiecka J, Podlasin R, et al. Tuberculosis and mycobacteriosis important opportunistic disease in AIDS patients. Przegl Epidemiol 2001; 55 Suppl 3: 117-125.

21. Kowalski J, Cholewinska G, Pyziak-Kowalska K, et al. The Spectrum of Malignancies among Adult HIV Cohort in Poland between 1995 and 2012: A Retrospective Analysis of 288 Cases. Contemp Oncol (Pozn) 2015; 19: 226-235.

22. Rosinska M, Marzec-Bogustawska A, Janiec J, et al. High percentage of recent HIV infection among HIV-positive individuals newly diagnosed at voluntary counseling and testing sites in Poland. AIDS Res Hum Retroviruses 2013; 29: 805-813.

23. Szostakowska M, Pogoda I, Ulanicka M, et al. Final Report. Evaluation of the National Programme for preventing HIV infection and providing interventions for people living with HIV/AIDS for years 2012-2015. Available at: https://politykaspoleczna.um.warszawa. pl/sites/politykaspoleczna.um.warszawa.pl/files/artykuly/zalaczniki/ewaluacja_programu_hiv_2012-2015.pdf

24. Horban A, et al. Polish AIDS Society Recommendations (PTN AIDS) 2016. Warsaw 2016; pp. 10-17.

25. Nakagawa F, Lodwick RK, Smith CJ, et al. Projected life expectancy of people with HIV according to timing of diagnosis. AIDS 2012; 26: 335-343.

26. EACS Guidelines, Version 8.0, October 2015; 21-25.

27. Ankiersztejn-Bartczak M, Firlag-Burkacka E, Czeszko-Paprocka H, et al. Factors responsible for incomplete linkage to care after HIV diagnosis: preliminary results from the Test and Keep in Care (TAK) project. HIV Med 2015; 16: 88-94.

28. Kowalska JD, Shepherd L, Ankiersztejn-Bartczak M, et al. Poor Linkage to Care Despite Significant Improvement in Access to Early cART in Central Poland - Data from Test and Keep in Care (TAK) Project. PLoS One 2016; 11: e0162739.

29. Camoni L, Raimondo M, Regine V, et al. Late presenters among persons with a new HIV diagnosis in Italy, 2010-2011. BMC Public Health $2013 ; 13: 281$.

30. 90-90-90 An ambitious treatment target to help end the AIDS epidemic. Available at: http://www.unaids.org/sites/default/files/media_asset/90-90-90_en_0.pdf

31. HIDES 2 (HIV Indicator Diseases Across Europe Study) 2011-2015. Available at: http://newsite.hiveurope.eu/Finalised-Projects/HIDES/ HIDES-2. 